\title{
Unintended pregnancy with implantable subdermal contraceptive device (Nexplanon): a case report
}

\begin{abstract}
Background: In 2006, Implanon (68 mg), a synthetic subcutaneous progestin etonogestrel eluting capsule, was approved for use in the U.S. to provide long-acting reversible contraception (LARC). The next generation LARC, Nexplanon $(68 \mathrm{mg})$, a progestin-only etonogestrel subdermal implant, was available in 2001. Currently, Nexplanon is advertised to provide $99 \%$ effective contraception, up to 4 years. Removal of the capsule will revert back to normal menstruation and fertility. Unlike Implanon, cases of unintended uterine pregnancy with proper and successful insertion have not been reported using Nexplanon.

Case: A 24-year-old female, G2P1001, with obstetrical history of one spontaneous vaginal delivery (2015), and gynecological history of low grade squamous intraepithelial lesion (LSIL) on Pap smear (2014), was found to have an intrauterine pregnancy while using Nexplanon. Initially, urine pregnancy testing assured that she was not pregnant before placement of the Nexplanon. Nexplanon was inserted $\sim 2$ months post-partum after the birth of her first child.17 months post-Nexplanon insertion, patient experienced irregular spotting, cramping, and reported positive home pregnancy test. Transvaginal ultrasound confirmed an intrauterine pregnancy at 9 weeks and 3 days. The Nexplanon implant was removed intact. Routine prenatal care was initiated and no issues were identified.

Conclusion: Newer LARCs provide an alternative to lifestyle adaptive contraception, with ease of use and compliance. Although LARCs prove to be more effective, known factors linked to human error during placement, cytochrome P450 induction, and BMI, have shown to decrease their efficacy, leading to failed contraception.
\end{abstract}

Keywords: Etonogestrel releasing implant, Implantable subdermal contraceptive, LARC, Nexplanon, Progestin-only hormone implant, Unintended pregnancy
Volume 9 Issue I - 2018

\author{
Shadi Rezai,' Neil D Patel, ${ }^{2}$ Alexander C \\ Hughes, ${ }^{2}$ Cynthia M Shumaker,' Bhavin \\ R Pandya,' Mon-Lai Cheung,' Basem Z \\ Bernaba,' Wendy J Knight,' Ray Mercado, ${ }^{4}$ \\ Takeko Takeshige, ${ }^{5}$ Paul N Fuller,' Cassandra \\ E Henderson ${ }^{3}$ \\ 'Department of Obstetrics and Gynecology, Southern California \\ Kaiser Permanente, USA \\ ${ }^{2}$ St Georges University, School of Medicine, Grenada \\ ${ }^{3}$ Department of Obstetrics and Gynecology, Lincoln Medical \\ and Mental Health Center, USA \\ 4Department of Obstetrics and Gynecology, Comprehensive \\ Medical Care of the Bronx, USA \\ ${ }^{5}$ Takeshige Medical, PO Box I2I, Edgewater, New Jersey, 07020, \\ USA
}

Correspondence: Shadi Rezai MD, Department of Obstetrics and Gynecology, Southern California Kaiser Permanente, Kern County, 1200 Discovery Drive, Bakersfield, California, 93309, USA,Tel 7185795000,Email shadi.rezai@kp.org

Received: December 05, 2017 | Published: January 31, 2018
Abbreviations: LARC, Long-Acting Reversible Contraception; SARC, Short-Acting Reversible Contraceptives; OCPs, Oral Contraceptive Pills; DMPA, Depot Medroxyprogesterone Acetate; MSD, Merck Sharp \& Dohme; HPO, Hypothalamic Pituitary Ovarian; BMI, Body Mass Index; LSIL, Low Grade Squamous Intraepithelial Lesion; AED, Antiepileptic Medication

\section{Introduction}

Implanon, an Etonogestrel capsule, containing $68 \mathrm{mg}$ of the synthetic progestin etonogestrel implant, was first introduced in Indonesia in 1998 and approved for use in the U.S. in 2006, as a subcutaneous hormone eluting capsule providing long-acting reversible contraception (LARC) ${ }^{1-4}$ Prior to the use of Implanon, shortacting reversible contraceptives (SARC) such as oral contraceptive pills (OCPs), birth control vaginal rings (example Nuvaring), transdermal contraceptive patches, depot medroxyprogesterone acetate (DMPA) injections (Depo-Provera) were prescribed. ${ }^{5}$ A recent study performed in 2016 compared SARC and LARC, affirming that LARC use provides superior contraception compared to SARC.$^{6,7}$ The LARC capsule is inserted by a nurse or physician, with an applicator, into the patient's nondominant upper arm. ${ }^{8}$ The implant manufacturer, Merck Sharp \& Dohme (MSD) stated that Implanon had shown to be more than $99 \%$ effective in clinical trials, and asserted that there is no contraceptive that is $100 \%$ effective. ${ }^{9}$ The mechanism of action of Implanon relies on the suppression of ovulation through hypothalamic pituitary ovarian (HPO) axis dysregulation and promotes thickening of the cervical mucus preventing fertilization. ${ }^{10}$ Between 1999 and 2011, over 1.4 million women used Implanon. ${ }^{1,2}$ An Australian study, published in 2005, found the Implanon failure rate to be 1 in $1000 .{ }^{11}$ This is the only comprehensive study of Implanon failure published. ${ }^{11}$

The next generation of Implanon, Nexplanon, approved in 2006, is inserted in the non-dominant upper arm. ${ }^{8}$ Nexplanon implants are reported to provide contraception for up to 4 years. ${ }^{8}$ If Nexplanon is implanted during the first 5 days of menstruation, it is immediately effective. ${ }^{12,13}$ However, if the capsule is implanted outside those 5 days, this contraceptive method will not be effective for one week and bridging contraception is required. ${ }^{12,13}$ Removal of the capsule will allow the menstrual cycle to resume normally. ${ }^{12,13}$ It has not previously been reported to be associated with an unintended uterine pregnancy with proper and successful insertion.

Contraindications for the implant include; known or suspected pregnancy, current or past history of thrombosis or thromboembolic disorders, liver tumors (benign or malignant), active liver disease, undiagnosed abnormal genital bleeding, known or suspected breast cancer, personal history of breast cancer, other progestin-sensitive cancer and allergies to the components of Nexplanon. ${ }^{12,13}$ Side effects of Nexplanon include; irregular menses, headache, vaginitis, breast pain, weight gain, acne, abdominal pain, and pharyngitis. ${ }^{12,13}$ Although Nexplanon is used to prevent pregnancy, if the implant fails, there is an increased risk for ectopic pregnancy. ${ }^{12}$ We present the first case of intrauterine pregnancy while using Nexplanon. ${ }^{14}$

\section{Case report}

This patient is a 24-year-old female, G2P1001, with past medical history of asthma controlled with albuterol, asthmatic bronchitis 
treated with amoxicillin, and iron deficiency anemia was found to have an intrauterine pregnancy while using Nexplanon. Obstetrical history is significant for one spontaneous vaginal delivery in 2015, and gynecological history includes low grade squamous intraepithelial lesion (LSIL) on pap smear in 2014, with no further abnormal pap smears. She had used Nexplanon before her first pregnancy with minimal side effects. Her body mass index (BMI) was $25.95 \mathrm{~kg} /$ $\mathrm{m} 2$. Vital signs were within normal limits throughout the course of treatment.

The patient requested Nexplanon for contraception after the birth of her first child. Nexplanon was inserted, as per manufacturer's instructions, approximately 2 months post-partum in the upper, nondominant arm (left arm) and verified by palpation. Urine pregnancy testing assured that she was not pregnant before placement of the Nexplanon. The expiration date was 2/2018. The patient returned 1 month later for a Nexplanon check presenting no new complaints. At that time, her method of contraception was condoms and the patient was breastfeeding.

The patient returned to the clinic 16 months later, 17 months post Nexplanon insertion, because she was experiencing irregular spotting, cramping, and a positive home pregnancy test. The patient could not recall her last menstrual period. In the clinic, the patient's $\beta$-hCG was $121,584 \mathrm{mIU} / \mathrm{ml}$. The transvaginal ultrasound confirmed an intrauterine pregnancy at 9 weeks and 3 days, with good fetal movement and good fetal heart motion (Figure 1). This was not a planned pregnancy, but the patient wished to continue to term. The Nexplanon implant was removed intact. Routine prenatal care was initiated and no issues were identified at the time of this case report.

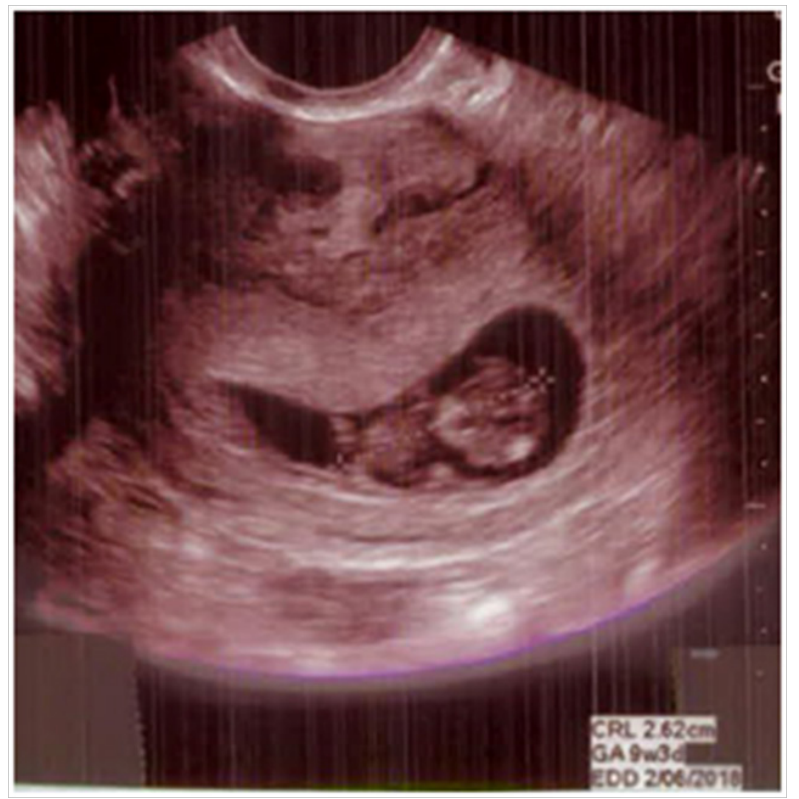

Figure I Transvaginal ultrasound on 7/7/I7 showed IUP at $93 / 7$ wks by CRL of $2.62 \mathrm{~cm}$, with EDD of $2 / 6 / 18$.

\section{Discussion and conclusion}

The case above describes a woman of reproductive age using Nexplanon as a LARC, which failed to provide contraception leading to IUP. Implanon, having been available since 1998, has reported hundreds of cases of unplanned pregnancy. The newer generation, Nexplanon which was used in this case, has been available since 2001, has not yet reported any cases of unplanned pregnancy. Merck, the manufacturer of Implanon and Nexplanon claim these are $99 \%$ effective; however, effectiveness can be reduced by factors such as improper insertion, device damage, drug interactions with Cytochrome P40 3A4 (CYP) inducers, and weight/BMI., ${ }^{92,13,15,16}$

The largest contributing factor to contraceptive failure results from improper capsule insertion. ${ }^{17}$ The effectiveness relies on correct subdermal capsule by a nurse or physician and proper post-insertion care. ${ }^{1,12,13}$ The device is placed in the patient's non-dominant arm, and may remain for up to 4 years. ${ }^{12,13}$ Correct insertion procedures are required for maximum efficacy. Therefore, it is critical that medical professionals be thoroughly trained to insert the device; ensuring the cartridge is loaded prior to application and unloaded after injection, then verified by palpation. ${ }^{12,13}$ After repeated failures, a study evaluated the causes of 200 contraceptive failures in 20,486 Australian women. ${ }^{17} 84$ of the 200 pregnancies were attributed to improper placement secondary to inadequate insertion training, in which the rod remained in the applicator or the rod falling out of the applicator, never actually being delivered. ${ }^{17}$ Further, the Australian study shows $46 / 200$ women were pregnant prior to implantation. ${ }^{17}$ As Merck has proclaimed previously, precise implantation during the menstrual cycle is crucial; the study showed 19/200 failures were due to untimely insertions. ${ }^{12,13,17}$

It is just as important to maintain the integrity of the implanted capsule as it is to properly insert the contraceptive device. Damage to the capsule itself should be thoroughly evaluated during implantation and removal. ${ }^{18}$ Cases have been reported of mis-positioning, migration, and damage to the capsule, altering the efficacy of the device. ${ }^{18}$ It is suspected that capsule damage can change the overall surface area, resulting in either too much, or too little progestin being released. ${ }^{18}$ The suspicion for failure due to misplacement and structural integrity in this patient is unlikely due to complete intact removal of the device from the original insertion site.

Another factor in LARC failure is drug-drug interactions. Estrogen and progesterone are metabolized by the hepatic enzyme CYP450 3A4 (CYP) system. CYP450 3A4 inducers have shown to decrease the efficacy of implanted LARCs, particularly, the use of antiepileptic medication (AED). ${ }^{19,20}$ Common hepatic enzyme AED inducers include phenytoin, phenobarbital, oxcarbazepine, topiramate, and carbamazepine..$^{19,21,22}$ Non-nucleoside reverse transcriptase inhibitors (efavirenz) and anti-tuberculosis medications (isoniazid) also have CYP induction properties..$^{23,24}$ These medications induce the CYP450 3A4 system to accelerate the metabolism of estrogen and progestin; thereby decreasing the available serum concentration of progestin..$^{19,21,22}$ In regards to this patient, concomitant drug use was not a factor in contraceptive failure, however, it should not be excluded from possible causes of LARC failure.

As with many other medications, a patient's body weight and BMI should be taken into consideration when initiating therapy or dosing. The current available LARC have one fixed dosing for all patients. Recent LARC failures have been attributed to a patient's BMI. ${ }^{25-27}$ The increased cell density requires a greater concentration of progesterone to be effective and is inversely related to the patient's body weight. .2,25-27 $^{2}$ Nexplanon product information includes a disclaimer outlining decreased LARC efficacy in overweight women. ${ }^{12,16}$ However, Merck has not performed specific studies in women who weighed above $130 \%$ of their ideal body weight to determine effectiveness in overweight women. ${ }^{12}$ It is also expected that progestin concentrations will decrease over time with use, combined with obesity, resulting in a significantly lower concentration of progestin in these women. ${ }^{12}$ In this case, the patient presented had a BMI of $25.95 \mathrm{~kg} / \mathrm{m} 2$ (normal 18.5-24.9, overweight 25-29.9). Since the patient's BMI calculates to 
the lower limit of overweight, it is unlikely that BMI significantly affected LARC efficacy.

As stated by the product label, removal of the capsule will return the body back to normal menstrual homeostasis, allowing for pregnancy. ${ }^{12,13}$ In patients using Implanon, return to a normal menstrual cyclical is expected within 30 months of implant removal. ${ }^{16,28}$ It is suspected that this patient had recommenced ovulation, likely due to subtherapeutic concentrations of progestin, inadequate for effective contraception; leading to conception despite her amenorrhea and LARC use.

In the case of contraceptive failure, it is crucial to thoroughly evaluate the progression of zygote implantation and maturation, just like any other pregnancy. Serious complications such as ectopic pregnancy have been linked to contraceptive therapy. Progestinonly contraceptive products, including LARC, have an established increased relative-risk for ectopic pregnancy, if fertilization does occur. ${ }^{12,13,29}$ The pathophysiology is thought to be related to decreased smooth muscle contraction of fallopian tubes. ${ }^{13,29}$ Fortunately, in our case the pregnancy was an ultrasound confirmed intra-uterine pregnancy, and Nexplanon was removed at 9 weeks gestation.

It is important to take into consideration the multitude of factors that influence the effectiveness of the treatment - prior to, and during use. It may be beneficial to regularly screen for progestin levels to ensure they are within therapeutic range. Furthermore, routine evaluation of the integrity, location, and positioning of the implant would promote optimal functioning.

New questions have been raised recently to investigate if other factors such as alcohol, thyroid hormone, liver disease, and immunological reaction to the implant have the capacity to affect contraceptive efficacy. It is crucial to evaluate which additional factors can alter contraceptive efficacy, not only to reassure the patient, but also to prevent the stress of an unplanned pregnancy.

\section{Acknowledgements}

The authors would like to thank Ms. Judith Wilkinson, Medical Librarian at Lincoln Medical and Mental Health Center Science Library for providing the reference articles.

\section{Conflicts of Interest}

The Authors did not report any potential conflicts of interest.

\section{References}

1. I got pregnant after my Implanon contraceptive implant failed. Mirror, UK. 2011.

2. Implanon: 600 pregnancies despite contraceptive implant. BBC News Health. 2011.

3. Senanayake P, Malcolm P. Atlas of Contraception. $2^{\text {nd }}$ (edn), Informa Healthcare, UK. 2008;pp.160.

4. Shoupe Donna, Daniel R Mishell. The Handbook of Contraception: A Guide for Practical Management. $2^{\text {nd }}($ edn), Humana Press, USA. 2015;pp.312.

5. Pillai S. Advice on short-acting contraception. PJ Online. 2012;p.1-4.

6. Hubacher D, Spector H, Monteith C, et al. Long-acting reversible contraceptive acceptability and unintended pregnancy among women presenting for short-acting methods: a randomized patient preference trial. Am J Obstet Gynecol. 2017;216(2):101-109.

7. Bhatia $\mathrm{P}$, Nangia S, Aggarwal S, et al. Implanon: subdermal single rod contraceptive implant, J Obstet Gynaecol India. 2011;61(4):422-425.
8. Birth Control Implant. Planned Parenthood Federation of America Inc. 2017.

9. Merck Sharpe, Dohme. Implanon ${ }^{\circledR}$ Product information. 2016.

10. Modi MN, Heitmann RJ, Armstrong AY. Unintended pregnancy and the role of long-acting reversible contraception. Expert Rev Obstet Gynecol. 2013;8(6):549-558.

11. Mansour D. Nexplanon $\left({ }^{\circledR}\right)$ : what Implanon $\left({ }^{\circledR}\right)$ did next. J Fam Plann Reprod Health Care. 2010;36(4):187-189.

12. Merck Sharp, Dohme BV. Nexplanon ${ }^{\circledR}$ (etonogestrel implant) Radiopaque Subdermal Use Only. 2011.

13. Nexplanon (etonogestrel implant) Risks and Side Effects. 2017.

14. Mommers E, Blum GF, Gent TG, et al. Nexplanon, a radiopaque etonogestrel implant in combination with a next-generation applicator: 3 -year results of a noncomparative multicenter trial. Am J Obstet Gynecol. 2012;207(5):388.

15. Mahoney D, Woolley J, Jadeja M. Implanon and Reports of Unintended Pregnancy: Media Interest in UK. 11th ISOP Annual Meeting 'Next Stop: Istanbul-Bridging the Continents!'Istanbul, Turkey. 2011;34(10):976.

16. Miller V. Pregnancy despite Implanon ${ }^{\circledR}$. Healthed Pty Ltd, Australia. 2017.

17. Harrison-Woolrych M, Hill R. Unintended pregnancies with the etonogestrel implant (Implanon): a case series from postmarketing experience in Australia. Contraception. 2005;71(4):306-308.

18. Elliman A. Removal of a fractured Nexplanon ${ }^{\circledR}$ : MSD response. $J$ Fam Plann Reprod Health Care. 2013;39(1):66-67.

19. Lange J, Teal S, Tocce K. Decreased efficacy of an etonogestrel implant in a woman on antiepileptic medications: a case report. J Med Case Rep. 2014;8:43.

20. Lazorwitz A, Davis A, Swartz M, et al. The effect of carbamazepine on etonogestrel concentrations in contraceptive implant users. Contraception. 2017;95(6):571-577.

21. Gaffield ME, Culwell KR, Lee CR. The use of hormonal contraception among women taking anticonvulsant therapy. Contraception. 2011;83(1):16-29.

22. Reddy DS. Clinical pharmacokinetic interactions between antiepileptic drugs and hormonal contraceptives. Expert Rev Clin Pharmacol. 2010;3(2):183-192.

23. Gbolade B. Failure of Implanon ${ }^{\circledR}$ on antituberculous therapy. Open Access J Contraception. 2010;1:103-105.

24. Leticee N, Viard JP, Yamgnane A, et al. Contraceptive failure of etonogestrel implant in patients treated with antiretrovirals including efavirenz. Contraception. 2012;85(4):425-427.

25. Lopez LM, Bernholc A, Chen M, et al. Hormonal contraceptives for contraception in overweight or obese women. Cochrane Database Syst Rev. 2016;(8):CD008452.

26. Trussell J, Aiken A. Effect of obesity on the effectiveness of hormonal contraceptives. Contraception. 2015;92(6):602.

27. Yamazaki M, Dwyer K, Sobhan M, et al. Effect of obesity on the effectiveness of hormonal contraceptives: an individual participant data meta-analysis. Contraception. 2015;92(5):445-452.

28. Mäkäräinen L, van Beek A, Tuomivaara L, et al. Ovarian function during the use of a single contraceptive implant: Implanon compared with Norplant. Fertil Steril. 1998;69(4):714-721.

29. Callahan R, Yacobson I, Halpern V, et al. Ectopic pregnancy with use of progestin-only injectables and contraceptive implants: a systematic review. Contraception. 2015;92(6):514-522. 